\title{
Analysis of serum HE4 levels in various histologic subtypes of epithelial ovarian cancer and other malignant tumors
}

\author{
Alexandra Blackman ${ }^{\mathrm{a}}$, Jessica Mitchell ${ }^{\mathrm{a}}$, Rachael Rowswell-Turner ${ }^{\mathrm{a}}$, Rakesh Singh ${ }^{\mathrm{a}}$, \\ Kyu Kwang Kim ${ }^{\mathrm{a}}$, Elizabeth Eklund ${ }^{\mathrm{b}}$, Steven Skates ${ }^{\mathrm{c}}$, Robert C. Bast ${ }^{\mathrm{d}}$, Geralyn Messerlian ${ }^{\mathrm{b}}$, \\ M. Craig Miller ${ }^{\mathrm{a}}$ and Richard G. Moore ${ }^{\mathrm{a}, *}$ \\ ${ }^{a}$ Division of Gynecologic Oncology, Department of Obstetrics and Gynecology, Wilmot Cancer \\ Institute, University of Rochester, Rochester, NY, USA \\ ${ }^{\mathrm{b}}$ Department of Pathology, Women and Infants Hospital, Brown University, RI, USA \\ ${ }^{\mathrm{c}}$ Massachusetts General Hospital, Harvard University, Boston, MA, USA \\ ${ }^{\mathrm{d}}$ Department of Experimental Therapeutics, University of Texas M.D. Anderson Cancer Center, \\ Houston, TX, USA
}

Received 14 July 2021

Accepted 4 November 2021

\begin{abstract}
.
BACKGROUND: The measurement of serum HE4 levels has emerged as a sensitive and specific biomarker for epithelial ovarian cancers (EOCs). However, serum levels in women diagnosed with various histologic subtypes of EOC and in women with metastatic non-ovarian primary malignancies have not been widely reported.

OBJECTIVE: The goal of this study was to identify how serum HE4 levels vary in women diagnosed with different histologic subtypes of EOC and non-ovarian malignancies.

METHODS: Data from six prospective pelvic mass clinical trials was combined and an evaluation of serum HE4 levels in women diagnosed with a malignancy was performed. For all patients, serum was obtained prior to surgery and final pathology, including primary tumor site, histologic subtype, grade and stage, were recorded. The mean, median, standard deviation, maximum, and minimum HE4 levels were determined for each group.

RESULTS: A total of 984 patients were included in this study, with the average patient age being 60 years old. There were 230 premenopausal and 754 postmenopausal patients. Serum HE4 levels were elevated ( $\geq 70.0 \mathrm{pMol})$ in $85 \%$ of EOCs, $40 \%$ of LMP tumors, $21 \%$ of non-EOCs (germ cell tumors), $25 \%$ of cervical cancers, and $47 \%$ of non-gynecologic metastatic cancers. Analysis of histologic subtypes revealed $90 \%(n=391)$ of serous, $85 \%(n=73)$ of endometrioid, $45 \%(n=42)$ of mucinous, $86 \%(n=51)$ of mixed tumors, and $69 \%(n=36)$ of clear cell tumors had elevated serum HE4 levels.

CONCLUSIONS: Serum HE4 levels are most often elevated in women with high grade serous and endometrioid EOCs, and though serum elevations are seen more often with advanced stage disease, HE4 is also often elevated in early stage disease and lower grade tumors.
\end{abstract}

Keywords: Serum HE4, biomarker, ovarian cancer

\footnotetext{
*Corresponding author: Richard G. Moore, MD, Department of Obstetrics and Gynecology, Wilmot Cancer Institute, University of Rochester Medical Center, Rochester, NY 14620, USA. Tel.: +1 585 4428020; Fax: +1 585 4428039; E-mail: Richard_Moore@URMC.Rochester.EDU. ORCID ID: 0000-0001-5598-5263
} 


\section{Introduction}

Ovarian cancer is the leading cause of death due to gynecologic malignancies and the fifth leading cause of cancer deaths in women in the United States [1]. World wide it is estamated there are 314,000 new ovarian cancers annually with 207,300 each year [2]. The term Epithelial Ovarian Cancer (EOC) in this report is used to encompass primary epithelial ovarian cancer, fallopian tube cancer and primary peritoneal cancers. The majority of EOCs are high-grade serous cancers $(70 \%)$ followed by endometrioid (10\%), clear cell $(10 \%)$, mucinous $(3 \%)$, and low-grade serous tumors $(<5 \%)$, with carcinosarcoma and undifferentiated carcinomas being rare. Epithelial ovarian cancers have been placed into two categories based on morphologic, molecular genetic features and prognostic factors [3]. Type 1 EOCs are made up of low grade serous, low grade endometrioid, clear cell, mucinous and transitional cell cancers, while Type 2 EOCs include high grade serous carcinoma, high grade endometrioid, undifferentiated carcinoma and carcinosarcoma. Type 2 serous EOCs typically have TP53 mutations in up to $80 \%$ of the tumors, whereas Type 1 low grade serous EOCs harbor KRAS, BRAF and ERBB2 mutations in up to $70 \%$ of the tumors. Recent data has called into question the origin of high-grade epithelial ovarian cancers, with the prevailing theory being that the majority are in fact arising from implanted cells of fallopian tube origin. To support this, precursor lesions for serous carcinoma have been identified in the fallopian tube and are labeled as serous tubal intraepithelial carcinomas, or STIC lesions. Additionally, clear cell and endometrioid malignancies have been shown to develop in the background of endometriosis $[4,5]$.

The WAP Four-Disulfide Core Domain 2 gene (WFDC2) has been identified as a commonly over expressed gene in EOC and encodes the secretory protiein human epididymis protein 4 (HE4). HE4 can be detected in the serum of patients diagnosed with EOC and has been cleared by the US FDA and other countries for the detection and monitoring ovarian cancers [6, 7]. HE4 has emerged as a useful predictive biomarker for EOC, and when combined with CA-125 testing, it improves the sensitivity and specificity for cancer detection amongst women with adnexal masses over that of CA-125 alone [7,8]. Like CA-125, serum HE4 levels are elevated in approximately 80\% of women with EOC, yet HE4 has been demonstrated to have increased specificity, as it is less frequently elevated in benign gynecologic conditions $[9,10]$. Serum HE4 levels have also been shown to rise with increasing grade, stage, and aggressiveness of EOC [11, 12]. As a result, HE4 is not only useful for assessing the risk of malignancy in women with adnexal masses, but also for monitoring treatment responses in women with EOC and facilitating the earlier detection of recurrence [13]. Additionally, the overexpression of HE4 in serous ovarian cancer tumors has been associated with tumorogenisis, proliferation, chemoresistance and decreased survival [14].

Historically, the treatment of epithelial ovarian cancer has been approached as a homogenous disease, but the evolving understanding of the molecular and histologic differences between subtypes has important implications for the development of more personalized treatment approaches. While cellular and tissue HE4 expression in various histologic subtypes of malignant tissue has been well documented via immunohistochemical staining, western blot and PCR analysis [15, 16], the differential expression of serum HE4 levels has not been thoroughly described in terms of histologic categories of various tumors. The largest study to date by Hertlein et al measured serum HE4 levels from 704 samples from both males and females [17]. However, this study only included 215 ovarian cancers and did not breakdown the tumors by histologic subtypes. Recently, HE4 overproduction has been shown to decrease the cellular immune response in the ovarian cancer tumor microenvironment and may be a targetable protein for treatment [18]. Because of the evolving role of HE4 in ovarian and other gynecologic cancers, it is important to clearly understand which of the various malignancies and histologic subtypes overexpress the WFDC2 gene and can be measured through serum HE4 levels in women with these malignancies. The goal of this study was to determine the differential expression 
and the measurement of serum HE4 levels in women with various histologic subtypes of EOC and other gynecologic and non-gynecologic malignancies.

\section{Methods}

This study includes data obtained from six prospective Institutional Review Board (IRB) approved clinical trials. This study was approved by the University of Rochester Medical Center, Wilmot Cancer Institute Institutional Review Board. A database was created by compiling de-identified data from the six clinical trials (BWH pilot study, MD Anderson pilot study, WIH pilot study, FDI-03 pelvic mass trial, FDI-15 pelvic mass trial, WIH-RS study, WIH-Ovarian Biomarker Trial). In each study, serum was obtained from patients within 30 days prior to surgery. Final pathologic diagnoses for each patient were determined by trained gynecologic oncology pathologists, and the primary tumor site, histologic subtype, grade, and stage were recorded for each patient. Demographic factors such as age and

Table 1

Study demographics

\begin{tabular}{|c|c|c|c|c|c|c|}
\hline \multirow[t]{2}{*}{ Clinical Trial } & \multirow[t]{2}{*}{$N$} & \multirow{2}{*}{$\begin{array}{c}\text { Average } \\
\text { Age (Range) }\end{array}$} & \multicolumn{2}{|c|}{ Pre-Menopausal } & \multicolumn{2}{|c|}{ Post-Menopausal } \\
\hline & & & $\%$ & $\begin{array}{c}\text { Average } \\
\text { Age (Range) }\end{array}$ & $\%$ & $\begin{array}{c}\text { Average } \\
\text { Age (Range) }\end{array}$ \\
\hline BWH Pilot Study & 166 & $54(20-86)$ & $36.1 \%$ & $44(20-55)$ & $63.9 \%$ & $59(46-86)$ \\
\hline MD Anderson Pilot & 112 & $61(19-88)$ & $20.5 \%$ & $43(19-51)$ & $79.5 \%$ & $65(52-88)$ \\
\hline WIH Pilot Study & 227 & $64(18-95)$ & $15.0 \%$ & $42(18-51)$ & $85.0 \%$ & $68(48-95)$ \\
\hline FDI-03 National Trial & 184 & $59(21-87)$ & $25.5 \%$ & $43(21-59)$ & $74.5 \%$ & $65(42-87)$ \\
\hline FDI-15 National Trial & 89 & $58(25-83)$ & $24.7 \%$ & $43(25-55)$ & $75.3 \%$ & $63(48-83)$ \\
\hline WIH - RS & 25 & $62(37-84)$ & $12.0 \%$ & $41(37-44)$ & $88.0 \%$ & $65(51-84)$ \\
\hline WIH-OBMT & 181 & $60(23-91)$ & $22.7 \%$ & $42(23-64)$ & $77.3 \%$ & $65(45-91)$ \\
\hline All Combined & 984 & $60(18-95)$ & $23.4 \%$ & $43(18-64)$ & $76.6 \%$ & $65(42-95)$ \\
\hline
\end{tabular}

\section{ALL PELVIC MASSES}

all Ovary Endometrial Cervical Uterine Sarcoma non-Gyn Metastatic

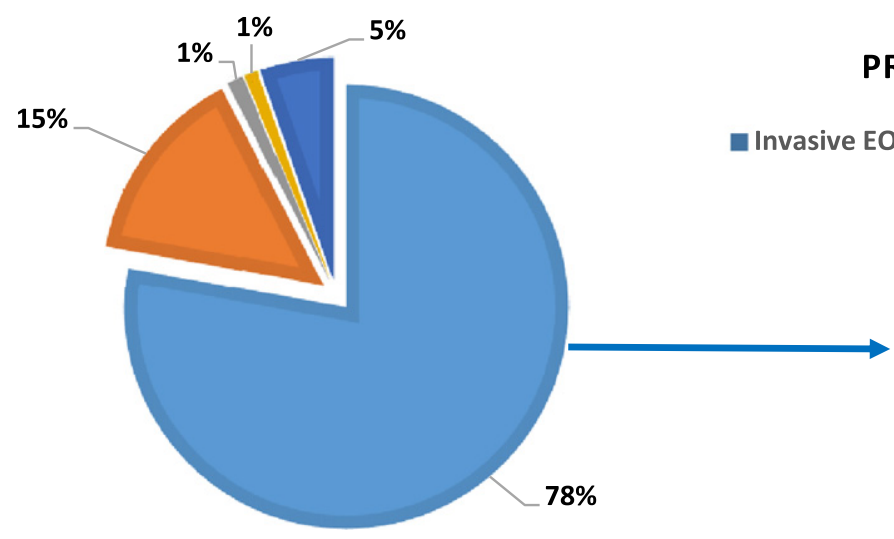

PRIMARY OVARY ONLY

Fig. 1. Distribution of cancer types as a percentage of all pelvic masses $(n=984)$. 
Table 2

Summary of HE4 for individual Primary malignancies and All Malignancies by menopausal status

\begin{tabular}{|c|c|c|c|c|c|c|c|c|c|c|c|c|c|c|c|}
\hline \multirow[t]{2}{*}{ Primary Site } & \multicolumn{5}{|c|}{ Pre-Menopausal } & \multicolumn{5}{|c|}{ Post-Menopausal } & \multicolumn{5}{|c|}{ All } \\
\hline & $N$ & Average \pm SD & Median & Min & Max & $N$ & Average \pm SD & Median & Min & Max & $N$ & Average \pm SD & Median & Min & Max \\
\hline Invasive EOC & 124 & $434.0 \pm 605.2$ & 213.4 & 17.2 & 4632.0 & 499 & $922.7 \pm 1873.0$ & 340.1 & 4.9 & 16991.0 & 623 & $825.5 \pm 1708.6$ & 318.1 & 4.9 & 16991.0 \\
\hline Borderline & 56 & $111.3 \pm 155.2$ & 62.7 & 20.1 & 880.3 & 73 & $94.2 \pm 109.2$ & 61.9 & 7.7 & 820.6 & 129 & $101.6 \pm 130.9$ & 62.6 & 7.7 & 880.3 \\
\hline $\begin{array}{l}\text { Germ } \\
\text { Cell \& SCST }\end{array}$ & 9 & $56.4 \pm 18.6$ & 53.5 & 29.4 & 85.5 & 5 & $63.3 \pm 42.9$ & 48.7 & 28.8 & 136.7 & 14 & $58.9 \pm 28.1$ & 51.1 & 28.8 & 136.7 \\
\hline $\begin{array}{l}\text { Uterine } \\
\text { (Endometrial) }\end{array}$ & 19 & $178.1 \pm 333.9$ & 45.3 & 25.7 & 1251.3 & 124 & $232.0 \pm 492.6$ & 95.2 & 15.3 & 4062.7 & 143 & $224.8 \pm 474.0$ & 84.6 & 15.3 & 4062.7 \\
\hline $\begin{array}{l}\text { Uterine } \\
\text { (Sarcoma) }\end{array}$ & 2 & $80.1 \pm 75.1$ & 80.1 & 27.0 & 133.2 & 8 & $73.3 \pm 28.6$ & 69.6 & 36.9 & 114.3 & 10 & $74.6 \pm 35.6$ & 69.6 & 27.0 & 133.2 \\
\hline Cervical & 4 & $48.9 \pm 29.3$ & 42.3 & 23.0 & 88.1 & 8 & $67.4 \pm 59.7$ & 41.3 & 23.3 & 184.2 & 12 & $61.2 \pm 50.8$ & 41.3 & 23.0 & 184.2 \\
\hline Colon & 10 & $108.5 \pm 109.0$ & 53.1 & 39.4 & 370.7 & 17 & $99.8 \pm 59.4$ & 81.7 & 33.9 & 214.8 & 27 & $103.0 \pm 79.4$ & 69.3 & 33.9 & 370.7 \\
\hline GI (Upper) & 5 & $48.2 \pm 26.6$ & 38.9 & 25.4 & 89.6 & 9 & $67.1 \pm 29.5$ & 57.3 & 28.9 & 114.0 & 14 & $60.4 \pm 29.0$ & 52.8 & 25.4 & 114.0 \\
\hline All Metastatic & 16 & $85.8 \pm 90.7$ & 50.7 & 25.4 & 370.7 & 37 & $102.1 \pm 79.8$ & 75.4 & 28.9 & 393.7 & 53 & $97.2 \pm 82.7$ & 62.9 & 25.4 & 393.7 \\
\hline $\begin{array}{l}\text { All Cancers + } \\
\text { LMP }\end{array}$ & 230 & $285.5 \pm 488.3$ & 88.4 & 17.2 & 4632.0 & 754 & $664.9 \pm 1579.0$ & 181.4 & 4.9 & 16991.0 & 984 & $576.2 \pm 1411.1$ & 153.2 & 4.9 & 16991.0 \\
\hline
\end{tabular}


menopausal status were also documented. Serum HE4 assays antibodies are manufactured by Fujirebio (Fujirebio Diagnostics, Malvern PA) and are used in the assay kits for the Abbott ARCHITEC i2000 platform (Abbot Diagnostics Inc., Chicago, IL, USA), the Fujirebio (CanAG) HE4 EIA test kit (Fujirebio Diagnostics, Gothenburg, Sweden) and the Lumiplse G-System HE4 assay (Fujirebio Diagnostics, Malvern, PA, USA). The Fujirebio manufactured kits for each platform have been standardized to the Fujirebio HE4 EIA kit, and the measurements are all comparable. Measurement of serum HE4 levels using the Roche and Abbott platforms compared to the Fujirebio HE4 EIA have been verified for harmonization and agreement of the automated methods versus the semi-automatedHE4 EIA method [19]. For each patient, one of the three HE4 assay platforms was used to determine serum HE4 levels prior to surgery, though the exact assay used varied by the clinical trial. An upper limit of normal threshold of $<70.0 \mathrm{pM}$ was chosen for this analysis. This cut point has previously been established and validated in several studies in healthy women and was used for this analysis [20,21]. The mean, median, standard deviation, maximum, and minimum HE4 levels were determined for each group. The expression levels of HE4 as a biomarker for malignancy was calculated for each category using a threshold of $\geq 70.0 \mathrm{pM}$. Student's t-testing was used to compare mean HE4 values between groups and Fisher's Exact test was used to compare proportions of subjects in the different groups with HE4 values below and above the cut point of $\geq 70.0 \mathrm{pM}$. One-way ANOVA with a Turnkey multiple com-

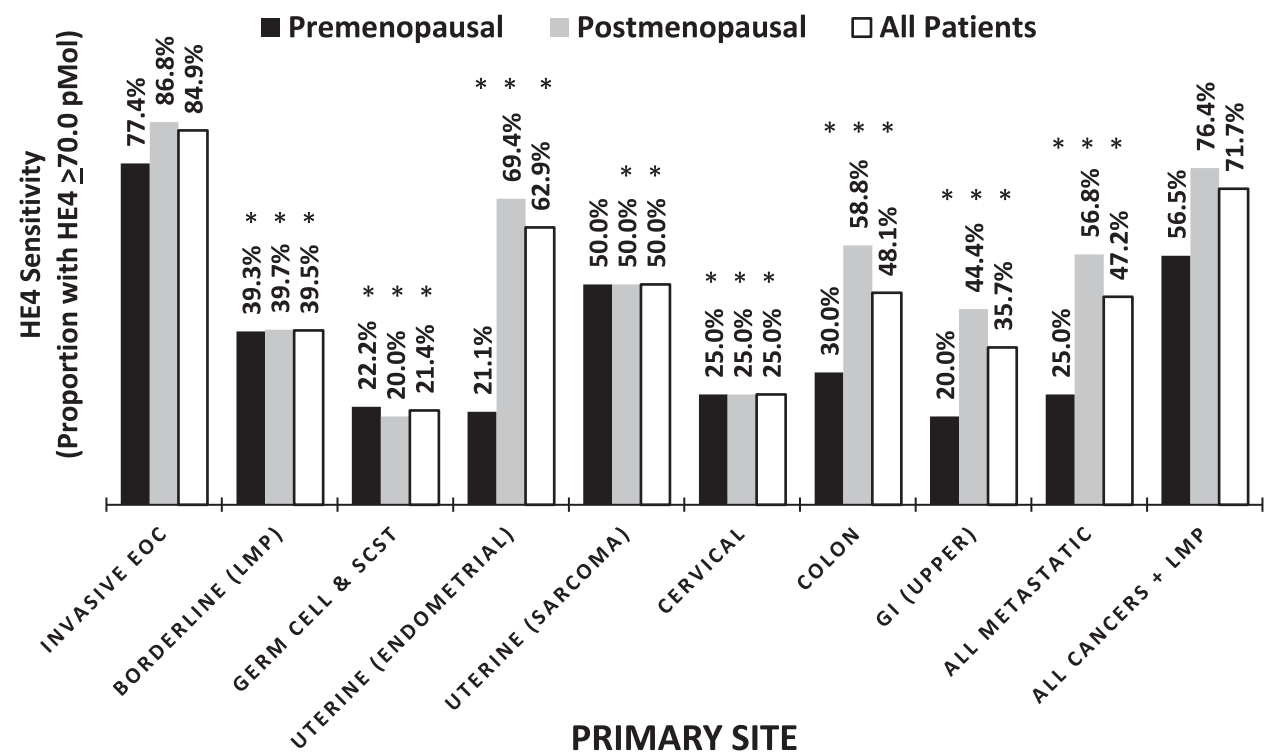

Fig. 2. Percentage of elevated HE4 across cancers, ANOVA $p$-value $=<0.0001 .{ }^{*}$ Significantly different when compared to All Invasive EOCs, $P<0.05$.

Table 3

Summary of HE4 for all invasive EOC histologic subtypes

\begin{tabular}{|c|c|c|c|c|c|c|c|c|c|c|c|c|c|c|c|}
\hline \multirow[t]{2}{*}{$\overline{\mathrm{EOC}}$} & \multicolumn{5}{|c|}{ Pre-Menopausal } & \multicolumn{5}{|c|}{ Post-Menopausal } & \multicolumn{5}{|c|}{ All } \\
\hline & $N$ & Average \pm SD & Median & Min & $\operatorname{Max}$ & $N$ & Average $\pm S D$ & Median & Min & Max & $N$ & Average $\pm S D$ & Median & Min & $\operatorname{Max}$ \\
\hline rous & 64 & $585.0 \pm 708.9$ & 386.0 & 39.9 & 4632.0 & 327 & $1021.9 \pm 1749.7$ & 447.3 & 4.9 & 16991.0 & 391 & $950.3 \pm 1632.9$ & 412.7 & 4.9 & 16991.0 \\
\hline ioid & 21 & $367.0 \pm 513.4$ & 167.5 & 18.6 & 1976.0 & 52 & $916.6 \pm 2365.3$ & 283.5 & 42.6 & 12800.0 & 73 & 758. & 269.6 & 18.6 & 12800.0 \\
\hline Clear Cell & 10 & $160.4 \pm 330.1$ & 58.1 & 17.2 & 1095.1 & 26 & \pm 270.0 & 5.2 & 30.0 & .5 & 36 & 6.7 & 108.7 & 17.2 & 1170.5 \\
\hline Mucinous & 15 & $87.4 \pm 100.3$ & 50.5 & 24.4 & 414.9 & 27 & \pm 204.3 & 97.0 & 12.7 & 1003.6 & 42 & 13 & 63.6 & 12.7 & 1003.6 \\
\hline Mixed & 10 & $441.7 \pm 551.4$ & 257.7 & 32.6 & 1853.8 & 41 & $1295.9 \pm 3217.3$ & 393.1 & 33.8 & 15146.6 & 51 & 907.3 & 336.8 & 32.6 & 15146.6 \\
\hline Rare $\mathrm{EOC}^{*}$ & 4 & $333.8 \pm 206.4$ & 326.3 & 90.5 & 592.0 & 26 & $551.9 \pm 539.7$ & 451.1 & 5.3 & 2095.2 & 30 & $522.8 \pm 511.0$ & 384.6 & 5.3 & 2095.2 \\
\hline
\end{tabular}

*Undifferentiated, Carcinosarcoma, neuroendocrine. 
parison post-test was also performed as appropriate. $P$-values $<0.05$ were considered to be statistically significant.

\section{Results}

A total of 984 patients with a malignancy were included in this study, of which 230 were premenopausal and 754 were postmenopausal women. The average patient age was 60 years (range 18 to 95). Serum HE4 levels were measured using the Fujirebio HE4 EIA assay in 896 samples, the ARCHITEC i2000 in 25 samples and Lumipulse G-Sytem in 63 samples. The number of patients from each trial included in this cohort as well as the average age and range of ages included in the study population are shown in Table 1 . There were 766 patients diagnosed with ovarian malignancies, including 623 invasive EOC, 129 borderline tumors and 14 germ cell or sex cord stromal tumors (SCST). Non-ovarian gynecologic primary malignancies included 143 uterine endometrial cancers, 12 cervical cancers and 10 uterine sarcomas. There were 53 metastatic non-gynecologic cancers, which included 27 colon, 14 small bowel and gastric, 5 pancreatic, 2 mesothelioma, 2 lymphoma and 1 each of kidney, lung, and breast cancers. The distribution of cancer types as a percentage of all pelvic masses in the study cohort are displayed in Fig. 1.

Evaluation of primary tumors revealed that HE4 was elevated $(\geq 70.0 \mathrm{pM})$ in $84.9 \%$ of all invasive EOC cases, with a lower proportion of the premenopausal women having elevated HE4 at the time of surgery compared to the postmenopausal women $(77.4 \%$ vs. $86.8 \%$, respectively, $p=0.012)$. In contrast, the serum levels of HE4 for other primary gynecologic and non-gynecologic malignancies was significantly lower $(p<0.05)$, indicating an increased expression of HE4 in more of the invasive EOCs compared with other primaries (Table 2, Fig. 2).

Examination of the histologic subtypes of invasive EOC revealed that HE4 was elevated in the majority of serous, endometrioid, and mixed tumors as well as in rare histologic subtypes (undifferentiated, carcinosarcoma and neuroendocrine), with levels $\geq 70.0 \mathrm{pM}$ in $90.0 \%, 84.9 \%, 86.3 \%$ and $90.0 \%$, respectively. A significantly smaller proportion of the patients with mucinous and clear cell carcinomas had elevated serum levels of HE4 compared to all other subtypes (Table 3 and Fig. 3,

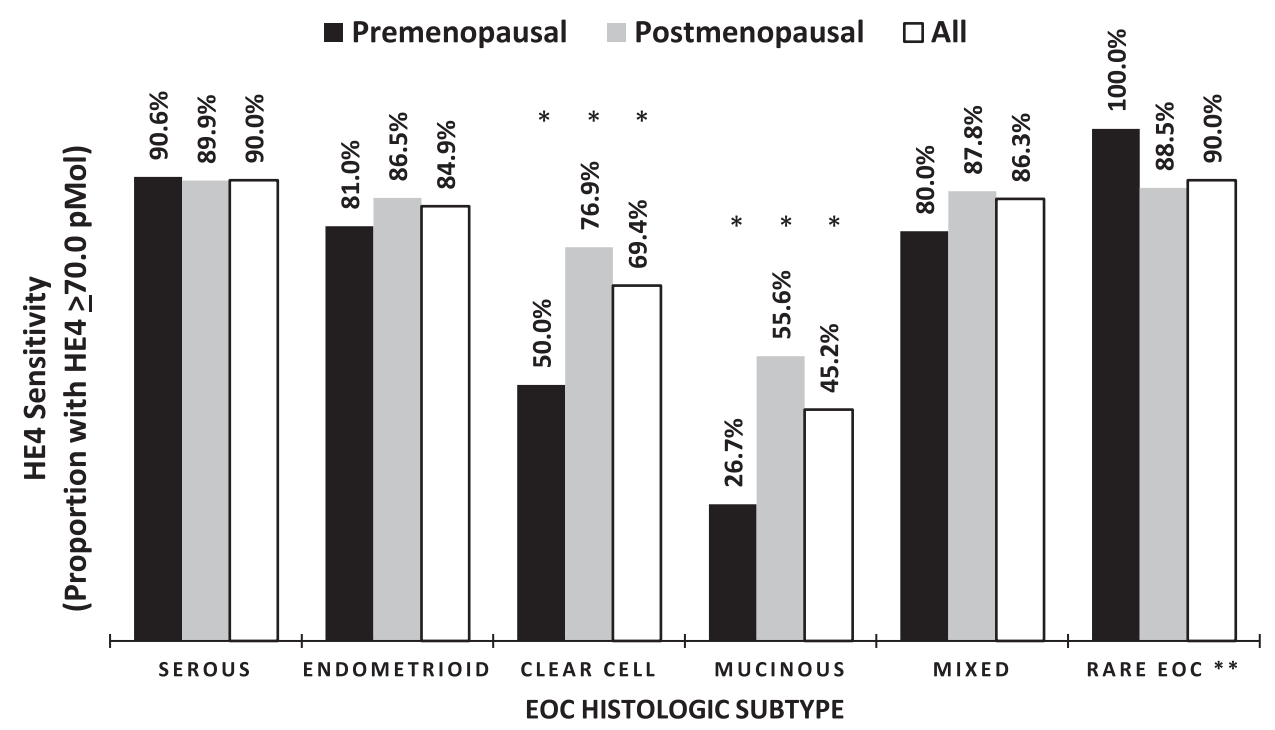

Fig. 3. Percentage of elevated HE4 across histologic subtypes of invasive EOC. ANOVA $p$-value $=0.0067$. ${ }^{*}$ Significantly different when compared to Serous EOCs, $P \leq 0.05$. ${ }^{* *}$ Rare EOC $=$ Undifferentiated, Carcinosarcoma, neuroendocrine. 
Table 4

Summary of HE4 by Stage and Grade of invasive EOC

\begin{tabular}{|c|c|c|c|c|c|c|c|c|c|c|c|c|c|c|c|}
\hline \multirow[t]{2}{*}{$\overline{\mathrm{EOC}}$} & \multicolumn{5}{|c|}{ Pre-Menopausal } & \multicolumn{5}{|c|}{ Post-Menopausal } & \multicolumn{5}{|c|}{ All } \\
\hline & $N$ & Average \pm SD & Median & Min & Max & $N$ & Average $\pm \mathrm{SD}$ & Median & Min & Max & $N$ & Average $\pm \mathrm{SD}$ & Median & Min & Max \\
\hline age I & 38 & 429.5 & 6.4 & 17.2 & 1976.0 & 88 & 296.9 & 9.9 & 5.3 & 1628.5 & 126 & 235 & 105.8 & 5.3 & 1976.0 \\
\hline ge II & 11 & .4 & 0 & 26.1 & 12 & 56 & 6 & .9 & 18.1 & 37 & 67 & & .4 & 18.1 & .0 \\
\hline age III & 66 & 632.3 & .1 & 39.9 & 4632.0 & 315 & $=2256.8$ & 576.5 & 6.7 & 16991.0 & 381 & 7.4 & 539.7 & 6.7 & 1.0 \\
\hline Stage IV & 6 & $1211.6 \pm 976.6$ & 1182.7 & 71.3 & 2796.0 & 37 & \pm 752.9 & 417.1 & 4.9 & 3189.0 & 43 & & 515.4 & 4.9 & 3189.0 \\
\hline rade 1 & 34 & $241.3 \pm 426.9$ & 96.3 & 17.2 & 1976.0 & 35 & $313.9 \pm 465.1$ & 140.2 & 32.1 & 2511.9 & 69 & $278.1 \pm 444.9$ & 115.9 & 17.2 & 2511.9 \\
\hline rade 2 & 15 & $330.7 \pm 283.4$ & 273.7 & 49.9 & 941.0 & 68 & $979.1 \pm 2063.5$ & 303.7 & 12.7 & 13250.0 & 83 & $861.9 \pm 1885.7$ & 283.0 & 12.7 & 13250.0 \\
\hline Grade 3 & 75 & $541.9 \pm 693.8$ & 349.2 & 26.0 & 4632.0 & 391 & $975.5 \pm 1920.2$ & 395.8 & 4.9 & 16991.0 & 466 & $905.8 \pm 1787.3$ & 387.5 & 4.9 & 16991.0 \\
\hline
\end{tabular}



Fig. 4. Percentage of elevated HE4 in relationship to Stage and Grade.

$p<0.05)$. These findings held true when taking into consideration menopausal status as well. ANOVA analysis demonstrated that serum levels are significantly different between the different histologic subtypes of invasive EOC $(p=0.007)$.

In addition to differential expression by histologic subtype, HE4 levels were stratified by cancer stage and grade, showing an increasing proportions of patients with elevated serum HE4 levels as both stage and grade increased (Table 4, Fig. 4). Serum HE4 levels increased with increasing FIGO stage in EOC, reflecting a correlation of HE4 expression and tumor burden. Serum HE4 levels were not significantly different between individual stages of EOC but was found to be significantly different between early and late-stage disease, with $65.8 \%$ of stage I-II cases $(n=193)$ having an elevated serum HE4 levels versus $93.6 \%$ of stage III-IV cases $(n=424)$ with elevated serum HE4 levels $(p<0.001)$. Serum HE4 was elevated in $60.9 \%, 84.3 \%$, and $88.4 \%$ of grade I $(n=69)$, II $(n=83)$, and III $(n=465)$ EOCs respectfully. Of note, the serum HE4 levels were similar for both pre and postmenopausal status across grade and stage (Fig. 4).

\section{Discussion}

The purpose of this study was to understand the differential expression of the serum tumor marker HE4 in women diagnosed with EOC and other malignancies. Serum HE4 was stratified across primary 
site of cancer, histologic subtype, grade, and stage of ovarian cancers. All serum samples were obtained prior to surgery and were correlated with histopathologic diagnosis. When comparing HE4 levels across histologic subtypes of EOC, increased serum HE4 levels were seen in serous, endometrioid and mixed histologic subtypes of EOC. Our findings also demonstrated that increasing serum HE4 levels correlate with advancing stage and grade of EOCs. The biomarker serum CA125 has been shown to be a prognostic indicator when highly elevated in women with EOC, and likewise, serum HE4 levels has also been shown to be an excellent biomarker for prognosis, monitoring response to treatment and detecting recurrent disease [22]. Additionally, both markers have been found to compliment one and another [7].

Serum HE4 measurements employed in the Risk of Malignancy Algorithm (ROMA) is useful as a tool for risk stratification in women with a pelvic mass, and therefore, understanding the expression of HE4 in various cancers is important $[7,8]$. HE4 is a relatively new clinical marker for the management of women with EOC and has been shown to be prognostic for stage, grade, disease recurrence, and more recently, chemoresistance [12, 23-25]. Here, we show that serum HE4 level does vary by cancer subtype, possibly explaining its strong prognostic impact. Additionally, HE4 over-expression has been associated with a more aggressive cancer phenotype, characterized by immune evasion, tumorigenesis, and cancer cell adhesion and motility [14, 26-28].

In the cancers evaluated in this analysis, mean levels of serum HE4 differed between histologic subtypes of EOC, with higher levels being more suggestive of serous or mixed EOC and lower levels more likely to be mucinous or clear cell on histologic examination. As our understanding of EOC has progressed, the heterogeneity of the disease has become more apparent. It is now widely known that EOC subtypes behave very differently. This difference in behavior is reflective of the varying genetic mutations that are found to be hallmarks for a particular subtype. As mentioned in the introduction, type 2 tumors, such as high-grade serous carcinoma, are more likely to harbor p53 mutations and carry a worse prognosis. In our study, patients with these types of tumors were more likely to exhibit higher serum levels of HE4. In 2013, Kristjansdottir and colleagues reported similar findings in a smaller cohort of 373 patients [29].

HE4 itself may prove to be a useful therapeutic target. Recent work focusing on HE4's function in cellular pathways has shown that its over-expression promotes proliferation, invasion, and metastases of cancer cells. HE4 has been implicated in diverse processes, from the EGFR-MAPK signaling pathway to steroid biosynthesis [30]. HE4 over-expression has also been shown to promote cisplatin resistance in ovarian cancer cells. In a 2014 study looking at ovarian cancer tumor cells and tumor growth in a mouse model, nuclear translocation of HE4 was linked with activity of VEGF and antisense targeting of HE4 suppressed tumor growth [14]. HE4 has also been shown in the tumor microenvironment to increase PD-L1 expression, inhibit CD8+ T cell infiltration, and decrease Natural Killer cell recruitment and differentiation of macrophages to the inactive $\mathrm{M} 2$ type, resulting in a decrease immune response in the tumor microenvironment [18]. Further investigation of the potential regulatory role of HE4 in these pathways may provide insight into why certain EOC subtypes respond poorly to standard therapies and provide new potential therapeutic targets to improve outcomes for patients. This study also highlighted an association between HE4 expression and more advanced grades and stages of EOC. This may be explained by recent studies that suggest a role for HE4 in cell adhesion and motility, which are essential to metastatic spread and the epithelial-to-mesenchymal transition [31]. Additionally, HE4 has been implicated in immune system evasion, and it is possible that targeting HE4 in this setting may restore the natural immune response and prevent or delay disease progression [27].

This is the largest study to date analyzing serum from 984 women to compare serum levels of HE4 amongst histologic subtypes of EOC as well as other gynecologic and non-gynecologic malignancies. Our data demonstrate that mean levels of serum HE4 differ across EOC subtypes, grade, and stage. Serum HE4 was elevated in a greater percentage of patients with serous, endometrioid and mixed 
subtypes. These findings provide further insight to the expression of HE4 in ovarian cancer tumors. The large number of cases included in the study is a major strength, and it is to our knowledge the largest study examining serum HE4 expression across pelvic tumors to date. Limitations of the study include the small number of other ovarian, gynecologic, and non-gynecologic malignancies, resulting in the inability to make a broader statement on malignancies. However, typically at least 10 cases are needed to determine expression in various tumors, and these numbers were reached in most instances of non-EOC tumors.

In summary, serum HE4 is elevated and over expressed for EOCs, particularly in patients facing advanced stage or high-grade disease. HE4 is not often expressed in non-EOCs or non-gynecologic metastatic pelvic tumors. HE4 expression shows variation among different histologic subtypes, with higher levels being found in serous, endometrioid and mixed subtypes.

\section{Acknowledgments}

The authors have no acknowledgments.

\section{Author contributions}

CONCEPTION: RGM

DATA CURATION: RS, KKK, EK, RCB, GM, MCM and RGM

ANALYSIS OF DATA: AB, JM, SS, GM, MCM and RGM

PREPARATION OF THE MANUSCRIPT: All authors

REVISION FOR IMPORTANT INTELLECTUAL CONTENT: All authors

SUPERVISION: RGM

\section{Conflict of interest}

Alexandra Blackman: no conflicts of interest.

Jesica Mitchel: no conflicts of interest.

Rakesh Singh: no conflicts of interest.

Kyu Kwang Kim: no conflicts of interest.

Steven Skates: Consulting for LUNGevity Foundation; Scientific Advisory Board for SISCAPA Assay Technologies and Mercy BioAnalytics.

Robert Bast: Royalties from Fujirebio Diagnostics Inc.

Geralyn Lambert-Messerlian: Research grants Fujirebio Diagnostics Inc.

M. Craig Miller: Consultant Fujirebio Diagnostics Inc.

Richard Moore: Consulting for Fujirebio Diagnostics Inc and Abcodia; Research grants Angle plc.

\section{References}

[1] Siegel RL, Miller KD, Fuchs HE, Jemal A. Cancer Statistics, 2021. CA: A Cancer Journal for Clinicians. 2021;71(1): 7-33.

[2] Ferlay J, Colombet M, Soerjomataram I, Parkin DM, Pineros M, Znaor A, et al. Cancer statistics for the year 2020: An overview. Int J Cancer. 2021.

[3] Kurman RJ, Shih Ie M. The origin and pathogenesis of epithelial ovarian cancer: a proposed unifying theory. Am J Surg Pathol. 2010;34(3):433-43. 
[4] Dubeau L. The cell of origin of ovarian epithelial tumours. Lancet Oncol. 2008;9(12):1191-7.

[5] Dubeau L, Drapkin R. Coming into focus: the nonovarian origins of ovarian cancer. Ann Oncol. 2013;24(Suppl 8):viii28viii35.

[6] Hellstrom I, Raycraft J, Hayden-Ledbetter M, Ledbetter JA, Schummer M, McIntosh M, et al. The HE4 (WFDC2) protein is a biomarker for ovarian carcinoma. Cancer Res. 2003;63(13):3695-700.

[7] Moore RG, Brown AK, Miller MC, Skates S, Allard WJ, Verch T, et al. The use of multiple novel tumor biomarkers for the detection of ovarian carcinoma in patients with a pelvic mass. Gynecol Oncol. 2008;108(2):402-8.

[8] Moore RG, Jabre-Raughley M, Brown AK, Robison KM, Miller MC, Allard WJ, et al. Comparison of a novel multiple marker assay vs the Risk of Malignancy Index for the prediction of epithelial ovarian cancer in patients with a pelvic mass. Am J Obstet Gynecol. 2010;203(3):228 e1-6.

[9] Molina R, Escudero JM, Auge JM, Filella X, Foj L, Torne A, et al. HE4 a novel tumour marker for ovarian cancer: comparison with CA 125 and ROMA algorithm in patients with gynaecological diseases. Tumour Biol. 2011;32(6):108795.

[10] Moore RG, Miller MC, Steinhoff MM, Skates SJ, Lu KH, Lambert-Messerlian G, et al. Serum HE4 levels are less frequently elevated than CA125 in women with benign gynecologic disorders. Am J Obstet Gynecol. 2012;206(4):351-8.

[11] Kalapotharakos G, Asciutto C, Henic E, Casslen B, Borgfeldt C. High preoperative blood levels of HE4 predicts poor prognosis in patients with ovarian cancer. Journal of Ovarian Research. 2012;5(1):20.

[12] Trudel D, Tetu B, Gregoire J, Plante M, Renaud MC, Bachvarov D, et al. Human epididymis protein 4 (HE4) and ovarian cancer prognosis. Gynecol Oncol. 2012;127(3):511-5.

[13] Gadducci A, Cosio S, Zola P, Landoni F, Maggino T, Sartori E. Surveillance procedures for patients treated for epithelial ovarian cancer: a review of the literature. Int J Gynecol Cancer. 2007;17(1):21-31.

[14] Moore RG, Hill EK, Horan T, Yano N, Kim K, MacLaughlan S, et al. HE4 (WFDC2) gene overexpression promotes ovarian tumor growth. Sci Rep. 2014;4:3574.

[15] Drapkin R, von Horsten HH, Lin Y, Mok SC, Crum CP, Welch WR, et al. Human epididymis protein 4 (HE4) is a secreted glycoprotein that is overexpressed by serous and endometrioid ovarian carcinomas. Cancer Res. 2005;65(6):2162-9.

[16] Galgano MT, Hampton GM, Frierson HF, Jr. Comprehensive analysis of HE4 expression in normal and malignant human tissues. Mod Pathol. 2006;19(6):847-53.

[17] Hertlein L, Stieber P, Kirschenhofer A, Krocker K, Nagel D, Lenhard M, et al. Human epididymis protein 4 (HE4) in benign and malignant diseases. Clin Chem Lab Med. 2012;50(12):2181-8.

[18] Rowswell-Turner RB, Singh RK, Urh A, Yano N, Kim KK, Khazan N, et al. HE4 Overexpression by Ovarian Cancer Promotes a Suppressive Tumor Immune Microenvironment and Enhanced Tumor and Macrophage PD-L1 Expression. J Immunol. 2021.

[19] Ferraro S, Borille S, Carnevale A, Frusciante E, Bassani N, Panteghini M. Verification of the harmonization of human epididymis protein 4 assays. Clin Chem Lab Med. 2016;54(10):1635-43.

[20] Karlsen NS, Karlsen MA, Hogdall CK, Hogdall EV. HE4 tissue expression and serum HE4 levels in healthy individuals and patients with benign or malignant tumors: a systematic review. Cancer epidemiology, biomarkers \& prevention : a publication of the American Association for Cancer Research, cosponsored by the American Society of Preventive Oncology. 2014;23(11):2285-95.

[21] Moore RG, Miller MC, Eklund EE, Lu KH, Bast RC, Jr., Lambert-Messerlian G. Serum levels of the ovarian cancer biomarker HE4 are decreased in pregnancy and increase with age. Am J Obstet Gynecol. 2012;206(4):349-7.

[22] Scaletta G, Plotti F, Luvero D, Capriglione S, Montera R, Miranda A, et al. The role of novel biomarker HE4 in the diagnosis, prognosis and follow-up of ovarian cancer: a systematic review. Expert Rev Anticancer Ther. 2017;17(9): $827-39$.

[23] Lu KH, Patterson AP, Wang L, Marquez RT, Atkinson EN, Baggerly KA, et al. Selection of potential markers for epithelial ovarian cancer with gene expression arrays and recursive descent partition analysis. Clin Cancer Res. 2004;10(10):3291-300.

[24] Paek J, Lee SH, Yim GW, Lee M, Kim YJ, Nam EJ, et al. Prognostic significance of human epididymis protein 4 in epithelial ovarian cancer. Eur J Obstet Gynecol Reprod Biol. 2011;158(2):338-42.

[25] Ribeiro JR, Schorl C, Yano N, Romano N, Kim KK, Singh RK, et al. HE4 promotes collateral resistance to cisplatin and paclitaxel in ovarian cancer cells. Journal of Ovarian Research. 2016;9(1):28.

[26] Lu R, Sun X, Xiao R, Zhou L, Gao X, Guo L. Human epididymis protein 4 (HE4) plays a key role in ovarian cancer cell adhesion and motility. Biochem Biophys Res Commun. 2012;419(2):274-80.

[27] James NE, Cantillo E, Oliver MT, Rowswell-Turner RB, Ribeiro JR, Kim KK, et al. HE4 suppresses the expression of osteopontin in mononuclear cells and compromises their cytotoxicity against ovarian cancer cells. Clin Exp Immunol. 2018;193(3):327-40. 
[28] James NE, Oliver MT, Ribeiro JR, Cantillo E, Rowswell-Turner RB, Kim KK, et al. Human Epididymis Secretory Protein 4 (HE4) Compromises Cytotoxic Mononuclear Cells via Inducing Dual Specificity Phosphatase 6. Front Pharmacol. 2019;10:216.

[29] Kristjansdottir B, Levan K, Partheen K, Sundfeldt K. Diagnostic performance of the biomarkers HE4 and CA125 in type I and type II epithelial ovarian cancer. Gynecol Oncol. 2013;131(1):52-8.

[30] Zhu L, Zhuang H, Wang H, Tan M, Schwab CL, Deng L, et al. Overexpression of HE4 (human epididymis protein 4) enhances proliferation, invasion and metastasis of ovarian cancer. Oncotarget. 2016;7(1):729-44.

[31] Ribeiro JR, Gaudet HM, Khan M, Schorl C, James NE, Oliver MT, et al. Human Epididymis Protein 4 Promotes Events Associated with Metastatic Ovarian Cancer via Regulation of the Extracelluar Matrix. Front Oncol. 2017;7:332. 